comparing sample sites oropharyngeal swabs have a significant lower load $(p<0.001)$, whereas urine samples have a significant higher load $(\mathrm{p}<0.001)$. Genital and anorectal swabs loads do not differ $(p=0.315)$. Lower bacterial load appears to be correlated with older patients.

Conclusion NG bacterial load is for a large part driven by sample site. Oropharyngeal NG infections are often asymptomatic which could be related to a lower bacterial load. However, the role of the observed load differences in transmission and symptoms should be addressed in future studies.

\section{P3.226 OROPHARYNGEAL TESTING AND POSITIVITY AT THE STI CLINIC IN THE PAST 5 YEARS; TESTING MORE AND FINDING EVEN MORE}

Van Liere Gafs, Dukers-Muijrers Nhtm, Hoebe Cjpa. Public Health Service South Limburg, Medical Microbiology Maastricht University Medical Centre, Geleen, The Netherlands

\subsection{6/sextrans-2017-053264.461}

Introduction Oropharyngeal Chlamydia trachomatis (CT) and Neisseria gonorrhoeae (NG) are not routinely tested for in STI clinic attendees. Although oropharyngeal infections are often asymptomatic, they contribute to transmission in the population. Routine systematic oropharyngeal testing was implemented in men who have sex with men (MSM) in 2010, and on behavioural indication in heterosexuals, but it is unknown whether this was successful in practice. Moreover, data on oropharyngeal testing and $\mathrm{CT} / \mathrm{NG}$ positivity in heterosexuals is limited.

Methods Men and women aged $>16$ years attending our STIclinic between 2009- 2015 were included $(n=47317)$. Specimens were tested using NAATs. Data were collected on demographics and sexual behaviour. Multivariable backward logistic regression analyses were used to test associations with oropharyngeal testing and oropharyngeal CT and NG. Tested determinants were age, sex, symptoms, number of sex partners and warned by (ex) partner.

Results Oropharyngeal testing in heterosexuals increased yearly from $13 \%$ in 2009 to $16 \%$ in 2015 (OR 1.3, 95\% CI 1.21.3). In MSM, testing increased from $88 \%$ to $98 \%$ (OR1.6, 95\% CI 1.5-1.8). Oropharyngeal CT positivity varied between $1.0 \%-1.6 \%$ in women $(54 / 4198)$, between $0 \%-1.3 \%$ in heterosexual men (13/2226) and between $0.8 \%-1.5 \%$ in MSM (79/ $8158)$, but was not associated with year of testing. Oropharyngeal NG positivity varied between $1.5 \%-3.4 \%$ in women (123/4201), between $0.6 \%-4.1 \%$ in heterosexual men (61/ 2226) and between $2.8 \%-6.4 \%$ in MSM (406/8156). Oropharyngeal NG positivity increased with year of testing in heterosexual men (OR 1.2, 95\% CI 1.01-1.4) and MSM (OR 1.2 95\% CI 1.1-1.2).

Conclusion Routine oropharyngeal testing in MSM was successfully implemented in practice. Oropharyngeal CT positivity remained stable after increased oropharyngeal testing in all groups, and in women also for oropharyngeal NG. In heterosexual men and MSM, increased oropharyngeal testing led to increased oropharyngeal NG positivity. This justifies routine oropharyngeal testing in MSM, and warrants careful monitoring in heterosexuals.

\section{P3.227 HIGH SUBSTANCE USE AND RISK FOR STI IN YOUNG HETEROSEXUALS AND MSM}

Van Liere Gafs, Hoebe Cjpa, Dukers-Muijrers Nhtm. Public Health Service South Limburg, Medical Microbiology Maastricht University Medical Centre, Geleen, The Netherlands

\subsection{6/sextrans-2017-053264.462}

Introduction Substance use to enhance sexual pleasure and performance is well known among men who have sex with men (MSM). Studies report a higher Chlamydia trachomatis (CT) and Neisseria gonorrhoeae (NG) prevalence among MSM who use substance before or during sex. Limited data exist on substance use in relation to CT and NG prevalence among young heterosexuals.

Methods CT and NG tested men and women aged $<25$ years attending our STI-clinic between 2010- 2015 were included $(n=3526)$. Specimens were tested using nucleic acid amplification tests. Data were collected on demographics, sexual behaviour, smoking, alcohol intake and drug use; marihuana, gamma hydroxy butyrate (GHB), ketamine, cocaine, heroin, speed, ecstasy and poppers. Univariable and multivariable backward logistic regression analyses were used to test associations between substance use and CT/NG. Tested determinants were age, symptoms, number of sex partners and warned by (ex) partner.

Results CT prevalence was $13.6 \%(\mathrm{n}=300)$ for women, $15.4 \%$ $(n=153)$ for heterosexual men and $10.6 \%(n=35)$ for MSM. For NG this was $1.0 \% \quad(n=23), 1.4 \% \quad(n=14)$, and $15.8 \%$ $(n=52)$ respectively. Substance use before or during sex varied between $26 \%-40 \%$ for drugs, $44 \%-67 \%$ for alcohol and $51 \%-64 \%$ for cigarette smoking. Among drug users, 39\%$45 \%$ used multiple drugs, most often marihuana (84\%), ecstasy (81\%) and cocaine (51\%). In young heterosexuals, smoking was independently associated with CT in women (OR 1.3 95\% CI 1.1-1.7), and ketamine use in men (OR 4.5, 95\% CI 1.6-12.7). For MSM, GHB use was independently associated with CT (OR 3.8, 95\% CI 1.2-12.2) and ketamine use with NG (OR 4.7, 95\% CI 1.3-16.9).

Conclusion Substance use before or during sex was reported often among young heterosexuals and MSM, and led to greater CT and NG (for MSM) risk. Different substance use was associated with different STI in different risk groups, therefore targeted care is an imperative. Prevention in STI clinics should include discussing drug use before or during sex, also in heterosexual youngsters.

\section{P3.228 STI PREVALENCE AMONG MALE VICTIMS OF A SEXUAL ASSUALT: DATA FROM 12 YEAR PERIOD, STI CLINIC AMSTERDAM, THE NETHERLANDS}

L Van Rooijen, Van Kempen, A Fewerda, MF Schim Van Der Loeff, HJC De Vries. Public Health Service (GGD) of Amsterdam, Amsterdam, The Netherlands

\subsection{6/sextrans-2017-053264.463}

Introduction Little is known about male sexual assault victims (SAV), frequency of health care seeking after such assaults and the prevalence of sexual transmitted infections (STI). The objective of this study was to assess the prevalence of STI among male SAV attending the STI clinic of Amsterdam, the Netherlands.

Methods In the electronic patient database, sexual assault (SA) is recorded as one of the reasons for visiting the clinic. We collected routine clinical data from the period 2005-2016. 
Characteristics and STI screening results of SAV and non-victims (NV) were compared. Backward multivariable logistic regression analysis was conducted to assess whether SAV was associated with STI positivity (chlamydia, gonorrhoea, infectious syphilis, infectious hepatitis B, and/or HIV).

Results Between 2005 and 2016 194,954 STI consultations were performed with male clients and in $135(0.07 \%)$ consultations SA was reported. In $92 \%$ of the assaults no condom was used. In $91 \%$ of cases the assailant was a male. Forensic examination was performed in $13 \%$ of the cases. Prior to the STI clinic consultation, in $19 \%$ an HIV test had been performed and $35 \%$ were vaccinated against hepatitis B. SAV were less often Dutch ( $54 \%$ vs. $63 \%$ in NV, p=0.027), the median age was 28 years (vs. 30 in $\mathrm{NV}, \mathrm{p}=0.20$ ), and $28 \%$ reported STI related complaints (vs. $34 \%$ in $\mathrm{NV}, \mathrm{p}=0.15$ ). In the 6 months preceding the STI clinic visit, $56 \%$ of the male victims reported homosexual contacts only (vs. $39 \%$ in $\mathrm{NV}$, $\mathrm{p}<0.001$ ). STI positivity was $12.6 \%$ in SAV and $18.4 \%$ in $\mathrm{NV}$ $(\mathrm{p}=0.080)$. In multivariable analysis being an SAV was associated with a lower risk of STI (OR $0.51 ; 95 \%$ CI $0.51-0.86$ ). Conclusion Over twelve years, 135 male clients reported an SA. The majority of the sexual assaults posed a risk to contract an STI (no condom use and male assailant). SAV had a significant lower risk to test STI positive than NV attending the STI clinic. As most victims were not tested for HIV, and did not receive a hepatitis B vaccination after the assault, STI clinics can play a key role in providing care to SAV including STI testing.

\section{P3.229 STI PREVALENCE AND FOLLOW-UP AMONG FEMALE VICTIMS OF A SEXUAL ASSAULT TESTED AT THE STI CLINIC IN AMSTERDAM, THE NETHERLANDS}

L Van Rooijen, Van Kempen, A Fewerda, MF Schim Van Der Loeff, HJC De Vries. Public Health Service (GGD) of Amsterdam, Amsterdam, The Netherlands

\subsection{6/sextrans-2017-053264.464}

Introduction During a sexual assault (SA), female victims may become infected with sexual transmitted infections (STI). Because of possibly high infection rates and low percentage returning for treatment, several STI clinics provide empiric antimicrobial therapy at the first consultation. The objective of this study was to assess the STI prevalence and follow-up of female sexual assault victims (SAV) at the STI clinic of Amsterdam, the Netherlands.

Methods In the electronic patient database, SA is recorded as one of the reasons for visiting the clinic. We collected routine clinical data from the period 2005-2016. Characteristics and STI screening results of SAV and non-victims (NV) were compared. Backward multivariable logistic regression analysis was conducted to assess whether SAV was associated with STI positivity (chlamydia, gonorrhoea, infectious syphilis, infectious hepatitis B, and/or HIV).

Results Between 2005 and 2016 166,808 STI consultations were performed with female clients and in 1066 consultations SA was reported. In $96 \%$ of the assaults no condom was used. All the assailants were male. Forensic examination was performed in $22 \%$ of the cases. Prior to the STI clinic consultation, in $10 \%$ an HIV test had been performed, $27 \%$ were vaccinated for hepatitis $\mathrm{B}$ and in $11 \%$ a pregnancy test was performed. SAV were less often Dutch $(60 \%$ vs. $68 \%$ in NV, $\mathrm{p}<0.001$ ), the median age was 24 years (vs. 24 in NV, $\mathrm{p}=0.003$ ) and $34 \%$ reported STI related complaints (vs. $24 \%$ in $\mathrm{NV}, \mathrm{p}<0.001)$. STI positivity was $11.7 \%$ in SAV and $11.8 \%$ in $\mathrm{NV}(\mathrm{p}=0.53)$. In the multivariable analysis being an SAV was not associated with STI (OR 0.99; 95\% CI 0.821.19). $91.3 \%$ of the SAV requiring antibiotics returned to the clinic.

Conclusion The STI positivity in female SAV was comparable to NV attending the STI clinic. The return rate for treatment was high and does not support empiric prophylactic antimicrobial therapy. As most victims were not tested for HIV, and did not receive a hepatitis $B$ vaccination after the assault, STI clinics can play a key role in providing care to SAV including STI testing.

\section{P3.230 DOUBLE TROUBLE: THE IMPACT OF LOW RISK PERCEPTION AND HIGH RISK SEXUAL BEHAVIOUR ON CHLAMYDIA TRANSMISSION}

${ }^{1}$ Van Wees Da, ${ }^{1} \mathrm{C}$ Den Daas, ${ }^{2}$ Kretzschmar Me, ${ }^{1} \mathrm{JC}$ Heijne. ${ }^{1}$ National Institute for Public Health And The Environment (RIVM), Bilthoven, The Netherlands; ${ }^{2} J u l i u s$ Centre For Health Sciences and Primary Care, University Medical Centre Utrecht, Utrecht, The Netherlands

\subsection{6/sextrans-2017-053264.465}

Introduction Transmission of Chlamydia trachomatis (chlamydia) is influenced by both sexual behaviour and psychological determinants (i.e., risk perception). However, mathematical models describing chlamydia transmission often consider only sexual behaviour. We explored the influence of incorporating risk perception in a mathematical model that estimated the impact of different testing scenarios on chlamydia prevalence. Methods We developed a pair compartmental model with a susceptible-infected-susceptible structure representing heterosexuals aged 16-26 years. Model parameters were informed by an online pilot study $(n=173)$ on sexual behaviour, psychological determinants, and chlamydia (re-) infections and will be updated with data from an ongoing longitudinal cohort study. The model population was divided in two sexual risk behaviour groups (based on the number of partners in the last year) and further divided in two risk perception groups (based on perceived risk for chlamydia). We compared the impact of an overall testing uptake of $20 \%$ per year on population chlamydia prevalence with different testing scenarios: 1) differential uptake among sexual behaviour groups (higher uptake in high sexual behaviour group) and additionally 2) differential uptake among risk perception groups (higher uptake in high risk perception groups).

Results Respondents with high sexual risk behaviour (SB) and low risk perception (RP) had the highest mean number of partners (high $\mathrm{SB} /$ low $\mathrm{RP}=5.3$, compared to high $\mathrm{SB} /$ high $\mathrm{RP}=5.0$, low $\mathrm{SB} /$ high $\mathrm{RP}=1.3$ and low $\mathrm{SB} /$ low $\mathrm{RP}=1.1$, $\mathrm{p}<0.001$ ), shortest mean duration of partnerships (high $\mathrm{SB} /$ low $\mathrm{RP}=153$ days, compared to high $\mathrm{SB} /$ high $\mathrm{RP}=233$, low $\mathrm{SB} /$ high $\mathrm{RP}=512$ and low $\mathrm{SB} /$ low $\mathrm{RP}=607$ days, $\mathrm{p}<0.001$ ) and the highest percentage of self-reported chlamydia infections in the past year (high $\mathrm{SB} /$ low $\mathrm{RP}=22.2 \%$, compared to high $\mathrm{SB} /$ high $\mathrm{RP}=14.3 \%$, low $\mathrm{SB} /$ high $\mathrm{RP}=3.1 \%$ and low $\mathrm{SB} /$ low $\mathrm{RP}=0 \%, \mathrm{p}=0.02$ ). Models that did not consider differential testing uptake among risk perception groups overestimated the impact of testing on chlamydia prevalence. This effect was largest in the high sexual behaviour/low risk perception group. Conclusion Mathematical models incorporating risk perception could improve the estimation of the impact of testing interventions on the prevalence of chlamydia in specific subgroups. 